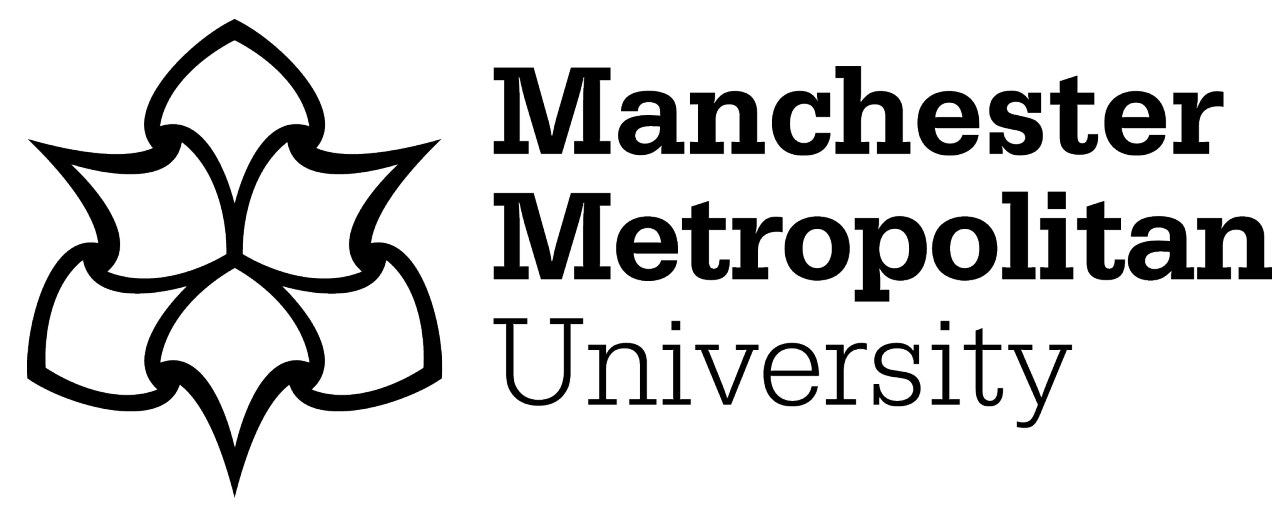

Rybakov, VV (2016) Non-transitive linear temporal logic and logical knowledge operations. Journal of Logic and Computation, 26 (3). pp. 945-958. ISSN 0955-792X

Downloaded from: https://e-space.mmu.ac.uk/465/

Version: Accepted Version

Publisher: Oxford University Press (OUP)

DOI: https://doi.org/10.1093/logcom/exv016

Please cite the published version 


\title{
Non-transitive linear temporal logic and logical knowledge operations
}

\author{
VLADIMIR V. RYBAKOV, School of Computing, Mathematics and DT, \\ Manchester Metropolitan University, John Dalton Building, Chester Street, \\ Manchester M1 5GD, UK. \\ E-mail:v.rybakov@mmu.ac.uk
}

\begin{abstract}
We study a linear temporal logic $\mathcal{L} \mathcal{T} \mathcal{L}_{N T}$ with non-transitive time (with NEXT and UNTIL) and possible interpretations for logical knowledge operations in this approach. We assume time to be non-transitive, linear and discrete, it is a major innovative part of our article. Motivation for our approach that time might be non-transitive and comments on possible interpretations of logical knowledge operations are given. The main result of Section 5 is a solution of the decidability problem for $\mathcal{L} \mathcal{T} \mathcal{L}_{N T}$, we find and describe in details the decision algorithm. In Section 6 we introduce non-transitive linear temporal logic $\mathcal{L} \mathcal{T} \mathcal{L}_{N T}(m)$ with uniform bound $(m)$ for non-transitivity. We compare it with standard linear temporal logic $\mathcal{L} \mathcal{T} \mathcal{L}$ and the logic $\mathcal{L} \mathcal{T} \mathcal{L}_{N T}-$ where non-transitivity has no upper bound-and show that $\mathcal{L} \mathcal{T} \mathcal{L}_{N T}$ may be approximated by logics $\mathcal{L} \mathcal{T} \mathcal{L}_{N T}(m)$. Concluding part of the article contains a list of open interesting problems.
\end{abstract}

Keywords: Linear temporal logic, non-transitive time, knowledge, logical knowledge operations, deciding algorithms.

\section{Introduction}

There are few concepts more interesting and mysterious as our conception of time. It was observed that temporal logic has important applications in formal verification, where it is used to state requirements of hardware or software systems. Historically, investigations of temporal logic in mathematical/philosophical logic based at modal systems was originated by Arthur Prior in late 1950s. Since then temporal logic has been (and is) a very active area in mathematical logic, information sciences, AI and CS (cf. e.g. Gabbay and Hodkinson [8-10]).

In particular, linear temporal logic $\mathcal{L T} \mathcal{L}$ (with Until and Next) is very useful instrument (cf. Manna, Pnueli [14, 15], Vardi [27, 28]; $\mathcal{L} \mathcal{T L}$ was used for analysing protocols of computations and checks of consistency, etc.). The main problems: decidability and satisfiability for $\mathcal{L} \mathcal{T} \mathcal{L}$ were the focus of investigations and were successfully resolved (cf. references above). The conception of knowledge, and especially the one implemented via a multi-agent approach is a popular area in Logic in Computer Science. Various aspects, including interaction and autonomy, effects of cooperation, etc. were investigated (cf. e.g. Wooldridge et al. [30-32], Lomuscio et al. [4, 13]). In particular, a multi-agent logic with distances was suggested and studied, satisfiability problem for it was solved (Rybakov et al. [22]); conception of Chance Discovery in multi-agents environment was considered (Rybakov [23, 24]); a logic modelling uncertainty via agents views was investigated (cf. McLean et al. [16]); representation of agents interaction (as a dual of the common knowledge-an elegant conception suggested and profoundly developed in Fagin et al. [6]) was suggested in Rybakov $[20,21]$.

Historically, the conception of common knowledge was formalized and technically developed in 1990s in a series of papers lately summarized in Fagin et al. [6]. In this approach, as the majority of ones accepted later for working with logical knowledge operations, the base was the agents knowledge

(C) The Author, 2015. Published by Oxford University Press. All rights reserved.

For Permissions, please email: journals.permissions@oup.com

doi:10.1093/logcom/exv016 


\section{Non-transitive LTL and logical knowledge operations}

and is represented as $S 5$-like modalities. Generally, the approach to model knowledge in terms of symbolic logic, probably, may be dated to the end of 1950. In 1962, Hintikka [12] wrote the book: Knowledge and Belief - very likely the first book to suggest using modalities to capture the semantics of knowledge.

In contemporary study, the field of knowledge representation and reasoning about knowledge in logical framework is a very popular area. Frequently, modal and multi-modal logics were used for formalizing agents reasoning. Such logics were suggested in Balbiani et al. [5], Vakarelov [29], Fagin et al. [6], Rybakov [17, 20]. Some up-to-date study of knowledge and beliefs expressed in terms of single-modal logic may be found in Halpern et al. [11]. The modern approach to knowledge frequently uses conception of justification in terms of epistemic logic (cf. e.g. Artemov et al. [1, 2]).

This article studies linear temporal logic $\mathcal{L} \mathcal{T} \mathcal{L}_{N T}$ with non-transitive time (with next $\mathbf{N}$ and until U) and possible interpretations for logical knowledge operations in this approach. We base our study on non-transitive time; it is the major innovative part of our article. We consider the logical Next operation and the operation until $\mathbf{U}$ as directed to past, and time to be discrete, linear, non-transitive and also directed to past. After the introduction, in Section 2 we recall preliminary information and notation concerning the standard (transitive) linear temporal logic $\mathcal{L} \mathcal{T} \mathcal{L}$. In Section 3, we give a motivation for our approach and explain why considering time to be non-transitive (intransitive) is reasonable, and why it gives plausible interpretations.

In Section 4, we formally introduce linear non-transitive temporal logic $\mathcal{L} \mathcal{T} \mathcal{L}_{N T}$ and prepare necessary technique for the main problem: decidability problem for $\mathcal{L} \mathcal{T} \mathcal{L}_{N T}$. In Section 5, we solve this problem: we show that $\mathcal{L} \mathcal{T} \mathcal{L}_{N T}$ is decidable and, hence, that the satisfiability problem for it is also decidable. We find and describe in details the deciding algorithm (note that since non-transitive time it is essentially more difficult than for the standard $\mathcal{L} \mathcal{T} \mathcal{L}$ itself). In Section 6, we introduce nontransitive linear temporal logic $\mathcal{L} \mathcal{T} \mathcal{L}_{N T}(m)$ with uniform bound $(m)$ for non-transitivity. We compare this logic with standard $\mathcal{L} \mathcal{T} \mathcal{L}$ and $\mathcal{L} \mathcal{T} \mathcal{L}_{N T}$ - where non-transitivity has no upper bound-and show that $\mathcal{L} \mathcal{T} \mathcal{L}_{N T}$ may be approximated by logics $\mathcal{L} \mathcal{T} \mathcal{L}_{N T}(m)$. We conclude the article by enumeration of some open interesting problems.

\section{Necessary preliminary information}

The main aim of our investigation is a new non-transitive version of the linear temporal logic $\mathcal{L} \mathcal{T}$ (motivation for non-transitivity will be given in a short separate section below). Therefore, we begin from a recall of notation and definitions concerning standard linear temporal logic $\mathcal{L} \mathcal{T}$. The reader familiar with $\mathcal{L} \mathcal{T} \mathcal{L}$ may skip this section and go straightforward to the next one.

The language of the Linear Temporal Logic $(\mathcal{L} \mathcal{T}$ in the sequel) extends the language of Boolean logic by the operations $\mathbf{N}$ (next) and $\mathbf{U}$ (until). The formulas of $\mathcal{L} \mathcal{T} \mathcal{L}$ are built up from a set Prop of atomic propositions (synonymously-propositional letters) and are closed under applications of Boolean operations, the unary operation $\mathbf{N}$ (next) and the binary operation $\mathbf{U}$ (until) (and yet, in some extended versions of $\mathcal{L} \mathcal{T} \mathcal{L}$, the binary operation $\mathbf{S}$ (since), and the unary operation $\mathbf{P r}$ - previous). The formula $\mathbf{N} \varphi$ has the meaning: the statement $\varphi$ holds in the next time point (state); the formula $\operatorname{Pr} \varphi$ means: the statement $\varphi$ was true at the immediate previous time point; the formula $\varphi \mathbf{U} \psi$ means: $\varphi$ holds until $\psi$ will be true (the formula $\varphi \mathbf{S} \psi$ means: $\varphi$ holds since $\psi$ has been true). Semantics for $\mathcal{L} \mathcal{T} \mathcal{L}$ consists of infinite transition systems (runs, computations); formally they are represented as linear Kripke structures based on the natural numbers.

The infinite linear Kripke structure is a quadruple $\mathcal{M}:=\langle\mathcal{N}, \leq, \operatorname{Next}, V\rangle$, where $\mathcal{N}$ is the set of all natural numbers (for some extended versions of $\mathcal{L} \mathcal{T} \mathcal{L}$ - the set of all integer numbers $Z$ ); $\leq$ is the 
standard order on $\mathcal{N}$, Next is the binary relation, where $a$ Next $b$ means $b$ is the number next to $a$. $V$ is a valuation of a subset $S$ of Prop. Hence, the valuation $V$ assigns truth values to elements (letters) of $S$. So, for any $p \in S, V(p) \subseteq \mathcal{N}, V(p)$ is the set of all numbers $n$ from $\mathcal{N}$ where the proposition $p$ is true (w.r.t. $V$ ).

All elements of $\mathcal{N}$ are possible states (worlds), $\leq$ is the transition relation (which is linear in our case), and $V$ can be interpreted as labelling of the states with atomic propositions. The triple $\langle\mathcal{N}, \leq$, Next $\rangle$ is a Kripke frame which we will denote for short by $\mathcal{N}$.

The truth values in any Kripke structure $\mathcal{M}$, can be extended from propositions of $S$ to arbitrary formulas constructed from these propositions as follows:

DEFINITION 1

Computational rules for logical operations:

- $\forall p \in \operatorname{Prop}(\mathcal{M}, a) \vdash_{V} p \Leftrightarrow a \in V(p)$;

- $(\mathcal{M}, a) \vdash_{V}(\varphi \wedge \psi) \Leftrightarrow(\mathcal{M}, a) \vdash_{V} \varphi \wedge(\mathcal{M}, a) \vdash_{V} \psi ;$

- $(\mathcal{M}, a) \Vdash_{V} \neg \varphi \Leftrightarrow \operatorname{not}\left[(\mathcal{M}, a) \Vdash_{V} \varphi\right]$;

- $(\mathcal{M}, a) \Vdash{ }_{V} \mathbf{N} \varphi \Leftrightarrow\left[\left[(a \operatorname{Next} b) \Rightarrow(\mathcal{M}, b) \Vdash{ }_{V} \varphi\right]\right]$;

- $(\mathcal{M}, a) \vdash_{V} \operatorname{Pr} \varphi \Leftrightarrow\left[\left[(b \operatorname{Next} a) \Rightarrow(\mathcal{M}, b) \Vdash{ }_{V} \varphi\right]\right]$;

- $(\mathcal{M}, a) \Vdash_{V}(\varphi \mathbf{U} \psi) \Leftrightarrow \exists b\left[(a \leq b) \wedge\left((\mathcal{M}, b) \vdash_{V} \psi\right) \wedge \forall c\left[(a \leq c<b) \Rightarrow(\mathcal{M}, c) \vdash_{V} \varphi\right]\right]$;

- $(\mathcal{M}, a) \vdash_{V}(\varphi \mathbf{S} \psi) \Leftrightarrow \exists b\left[(b \leq a) \wedge\left((\mathcal{M}, b) \Vdash_{V} \psi\right) \wedge \forall c\left[(a \leq c<b) \Rightarrow(\mathcal{M}, c) \vdash_{V} \varphi\right]\right]$.

For a Kripke structure $\mathcal{M}:=\langle\mathcal{N}, \leq$, Next,$V\rangle$ and a formula $\varphi$ with letters from the domain of $V$, we say $\varphi$ is valid in $\mathcal{M}$ (denotation $-\mathcal{M} \Vdash \varphi)$ if, for any $b$ of $\mathcal{M}(b \in \mathcal{N})$, the formula $\varphi$ is true at $b$ (denotation: $(\mathcal{M}, b) \Vdash V \varphi)$.

The linear temporal logic $\mathcal{L} \mathcal{T} \mathcal{L}$ is the set of all formulas which are valid in all infinite temporal linear Kripke structures $\mathcal{M}$ based on $\mathcal{N}$ with standard $\leq$ and Next.

The modal operations $\square$ (necessary) and $\diamond$ (possible) might be defined via temporal operations as follows: $\diamond p:=\top \mathbf{U} p, \square p:=\neg \diamond \neg p$.

Now we are supplied with the necessary definitions and notation concerning linear temporal logic and we may start our informal discussion. We go to motivation of our approach with nontransitive time and modelling logical knowledge operations. This approach is actually based on ideas suggested in our preprints $[25,26]$, and here we extend this approach and give complete proofs.

\section{Informal motivation, discussion, what is knowledge in perspective of time}

It is easy to accept that the knowledge is not absolute and depends on the opinions of individuals (agents) (who accept a statement as safely true and reliable) and as well on what we (they) actually consider as true knowledge. We, first, would like to look at it via temporal perspective. Some evident trivial observations are that

(i) Human beings remember (at least some) past;

(ii) They do not know future at all (though they can surmise what will happen in immediate proximity time points);

(iii) Individual memory tells to us that the time in the past was linear (at least our perceptions do). 


\section{Non-transitive LTL and logical knowledge operations}

Therefore, it looks meaningful to look for interpretations of knowledge in linear temporal logic with accessibility relations and Next directed actually to past. We now discuss with examples several approaches to define the logical operations responsible for knowledge. Here we will use the unary logical operations $K_{i}$ with meaning - it is a logical knowledge operation. (Below we will consider models for $\mathcal{L} \mathcal{T} \mathcal{L}$ with interpretation that $N e x t$ and time accessibility relation are actually directed to past, so $\leq$ means- to be earlier.)

(i) Simple approach: when knowledge was discovered once and since then it always seen to be true:

$$
\begin{aligned}
\left.(N, a) \Vdash_{V} K_{1} \varphi \Leftrightarrow \exists b\left[(N, b+1) \nVdash_{V} \varphi\right) \wedge(a \leq b) \wedge(N, b) \Vdash{ }_{V} \varphi\right) \wedge \\
\left.\forall c\left[(a \leq c<b) \Rightarrow(N, c) \vdash_{V} \varphi\right]\right] .
\end{aligned}
$$

From first glance, it is a rather plausible interpretation. As bigger $b$ will be, as it would be most reasonable to consider $\varphi$ as a knowledge. But for $a=b$ this definition actually says to us nothing, this definition then admits one-day knowledge, which is definitely not good.

(ii) Rigid approach from temporal logic: knowledge if always was true:

$$
(N, a) \Vdash_{V} K_{2} \varphi \Leftrightarrow(N, a) \Vdash{ }_{V} \neg(\top \mathbf{U} \neg \varphi) .
$$

That is fine, though it is too rigid - it assumes that we know all past (and besides it does not admit that knowledge was obtained only since a particular time point).

(iii) Knowledge since parameterizing facts:

$$
(N, a) \vdash_{V} K_{\psi} \varphi \Leftrightarrow(N, a) \vdash_{V} \varphi \mathbf{U} \psi .
$$

This means $\varphi$ has the stable truth value-true, since some event happened in past (which is modelled now by $\psi$ to be true at a state). Thus, as soon as $\psi$ happened to be true in past, $\varphi$ always held true until now. Here we use standard until. The formula $\psi$ may have any desirable value, so, we obtain knowledge since $\psi$.

(iv) Approach: via agents knowledge as voted truth for the valuation:

This is very well established area, cf. the book Fagin et al. [6] and more contemporary publications e.g.- Rybakov [17, 20].

But, we would like to look at it from an another standpoint. Earlier knowledge operations (agents knowledge) were just unary logical operations $K_{i}$ interpreted as $S 5$-modalities, and knowledge operations were introduced via the vote of agents, etc.

We would like to suggest here somewhat very simple but anyway rather fundamental and it seems new. We assume that all agents have their own valuations at the frame $N$. That is we have $n$-much agents, and $n$-much valuations $V_{i}$ and, as earlier, the truth values w.r.t. $V_{i}$ of any propositional letter $p_{j}$ at any world $a \in N$. From an applications viewpoint, $V_{i}$ correspond to agents information about truth of statements $p_{j}$ (statements in this information may be different, differ on true/false). So, $V_{i}$ is just individual information.

How the information can be turned into local knowledge? One way is the voted value of truth: we consider a new valuation $V$, w.r.t. which $p_{i}$ is true at $a$ if majority (with chosen confidence, we may use approach from fuzzy logic if we wish), biggest part of agents, believes that $p_{i}$ is true at $a$. Then we obtain a model with a single (standard) valuation $V$, as earlier. Next, we can apply any of the known approaches. And knowledge may be interpreted in many ways, in particular, as it was offered here above. 
But then, simultaneously, we also may consider all old individual truth valuations $V_{i}$ for all composed formulas $\varphi$ (in a standard manner) and definitions for knowledge operations for any agent $i$ (they may be different), together with general knowledge operations (accepted by all/majority agents) that depend on achieved earlier (as above) global valuation $V$ on propositional statements. Of course, we may use much more temporal features, for example:

(v) Approach: via agents knowledge as resolution at evaluation state.

Here we suggest a way starting similar as in the case (iv) above until the introduction of different valuations $V_{i}$ of agent truth values for letters coding truth of statements. We now suggest

$$
(N, a) \Vdash_{V} K \varphi \Leftrightarrow \forall i\left[(N, a) \vdash_{V_{i}} \diamond \varphi \wedge \square[\neg \varphi \rightarrow \mathbf{N} \neg \varphi]\right.
$$

and

$$
(N, a) \Vdash V_{i} K \varphi \Leftrightarrow \forall i\left[(N, a) \Vdash V_{i} \diamond \varphi \wedge \square[\neg \varphi \rightarrow \mathbf{N} \neg \varphi]\right.
$$

Thus, in this case, we will allow usage of nested knowledge operations for $K$ in formulas for any truth valuation $V_{i}$ of any agent $i$ and also for the global truth valuation $V$. The decision procedures (for the logics based at this approach) are not known nowadays. We think that to resolve it is an interesting open problem.

Summarizing these observations, we think that the linear temporal logic is a very promising instrument for determination and elicitation of logical knowledge. In the sequel, our approach for various kind of logical knowledge operations will be based on an assumption that knowledge is a true fact, which observed and widely acknowledged to be true in past for reasonable time, and remained always true until now. However, we would like to alleviate the request for time to be transitive (and then to base our approach on a suggested modification).

\section{Why Time might be Non-Transitive.}

VIEW (I). Time in individual perception: time in past has been as much as I remember.

The option explains itself very well. An agent today may not remember what he/she/it remembered some years ago. Here we do not make any reference to truth or knowledge, only to individual perception, ability to remember events. Though, it might be that what was truth and knowledge earlier is not anymore today.

VIEW (II). Computational view. Inspections of protocols for computations are limited by time resources and have non-uniform length (yet, in any point of inspection, verification may refer to stored old protocols). Therefore, if we interpret our models as the ones reflecting verification of computations, the amount of records for past inspections of protocols is finite, limited. And not all of them might be inspected in the given time point.

VIEW (III). Agents-admins view. We may consider states (worlds of our model) as checkpoints of admins (agents) for the inspection of recorded states of the network in the past. Any admin has allowed amount of inspections for previous states, but only within the areas of its(his/her) responsibility (by security or another reasons). So, the accessibility to past records in time is not transitive again.

VIEW (IV). Agents-users view. If we consider the states of the models as the content of web pages available for users, and any web link as the accessibility relation, then starting from any web page user may achieve, using links in hypertext(s) some available by links web sites, etc. The latter ones may have web links that are available only for individuals possessing passwords for accessibility. 


\section{Non-transitive LTL and logical knowledge operations}

And users having password may continue web surf, etc. Clearly that in this approach, web browsing looks as non-transitive relation. Here, if we interpret web browsing as time-steps, the accessibility is intransitive.

VIEW (v). View on time in past for collecting knowledge. In human perception, only some finite intervals of time in past are available to individuals to inspect evens and to record knowledge collected to current time state. The time is past in our feelings looks as linear and any individual has only a finite amount of memory to remember information and events. There, in past, at foremost available (remembered) time point, individuals again had a remembered interval of time with collected information, and so forth. So, the time in past, generally speaking, looks as not transitive form viewpoint of extending knowledge (since transition of the one from past to future might lose some)

VIEW (VI). View in past for individuals as agents with opposition. Here the comment is similar to the case (iv) above, but we may consider the knowledge as the collection of facts which about only the majority (not compulsory all) of experts (agents) have affirmative positive opinion. And, in past time, the voted opinion of experts about facts could be different at distinct time points. Besides the time intervals remembered by experts might be very diverse (for distinct experts in past). Therefore, in this approach the time relation again looks as non-transitive from viewpoint of safe collection of information.

Now, being based on observations above about knowledge and non-transitivity of time, we introduce the following non-transitive linear models for time.

\section{Linear temporal logic based at non-transitive time, preparatory (preliminary) technique}

We start from a precise definition of the logic itself and then we explain how to interpret ideas about definitions of logical knowledge operations from above in such approach.

DEFINITION 2

$A$ linear non-transitive possible-worlds frame is

$$
\mathcal{F}:=\left\langle N, \leq \text { Next },\left(\bigcup_{i \in N}\left\{R_{i}\right\}\right)\right\rangle,
$$

where the set of all natural numbers $N$ is the union of the intervals $\left[i, m_{i}\right]$, where any $i$ is a member of a fixed for this frame set $X$ of natural numbers, $m_{i}$ is strongly bigger than $i$ and belongs to $X$. Besides, any two different $\left(i, m_{i}\right)$ have empty intersection. For any $i$ from $X$ and $j$ from $\left[i, m_{i}\right], R_{j}$ is the standard linear order on $\left[j, m_{i}\right]$. We fix notation $t(i)=m_{i}$ for any $i$ from $X$; and $(a$ Next $b)$ iff $(b=a+1)$.

Notice that we consider $\left(\bigcup_{i \in N}\left\{R_{i}\right\}\right)$ as not a binary relation, but as the infinite countable set of finite binary relations $R_{i}$. As earlier, we may define a model $\mathcal{M}$ on $\mathcal{F}$ by introducing a valuation $V$ on $\mathcal{F}$ and extend it on all formulas as earlier, but for formulas of sort $\varphi \mathbf{U} \psi$ we define the truth value as follows:

Definition 3

For any $a \in N$ :

$$
\begin{gathered}
(\mathcal{M}, a) \vdash_{V}(\varphi \mathbf{U} \psi) \Leftrightarrow \\
\exists b\left[\left(a R_{a} b\right) \wedge\left((\mathcal{M}, b) \vdash_{V} \psi\right) \wedge \forall c\left[(a \leq c<b) \Rightarrow(\mathcal{M}, c) \Vdash_{V} \varphi\right]\right] ; \\
(\mathcal{M}, a) \vdash_{V} \mathbf{N} \varphi \Leftrightarrow\left[(a \operatorname{Next} b) \Rightarrow(\mathcal{M}, b) \vdash_{V} \varphi\right] .
\end{gathered}
$$


DEFINITION 4

The logic $\mathcal{L} \mathcal{T} \mathcal{L}_{N T}$ is the set of all formulas which are valid in any model $\mathcal{M}$ with any valuation.

A short comparison of this logic with standard $\mathcal{L} \mathcal{T L}$ will be given in Section 6. The relation $\left\langle\bigcup_{i \in N}\left\{R_{i}\right\}\right\rangle$ is evidently non-transitive, though any $R_{i}$ is linear and transitive. Its action (more precisely - the whole interval $\left[i, m_{i}\right]$ itself) may be interpreted as the interval of time which agent $i$ remember. In any time point $i+k$ it might be the new agent, or the same as the old (previous) one-just those who inspect.

Thus, we may interpret this approach as $i \in N$ is a time point and $\left[i, m_{i}\right]$ is the time interval available for the agent responsible for verification/reasoning in the time point $i$. The name of the agent in this case is anonymous-those one who is responsible for $i$.

Being based at this interpretation, we may consider interpretations of various aspects of knowledge discussed above and their extended versions.

EXAMPLES

$$
(\mathcal{M}, a) \Vdash_{V} K \varphi \Leftrightarrow(\mathcal{M}, a) \Vdash_{V} \varphi \mathbf{U}\left[\left[\mathbf{N}^{m+1} \neg \varphi\right] \wedge\left[\mathbf{N}^{m} \varphi\right]\right] .
$$

Here $K$ acts to say that knowledge coded by $\varphi$ has been achieved only $m$ 'years' ago and holds true since then. This example works even in the linear temporal logic $\mathcal{L} \mathcal{T} \mathcal{L}$ itself.

$$
(\mathcal{M}, a) \Vdash_{V} K_{1} \varphi \Leftrightarrow(\mathcal{M}, a) \Vdash_{V} \square \neg \varphi \wedge \diamond(\neg \varphi \wedge \mathbf{N}(K \varphi)) .
$$

Now $K_{1}$ determines that $\varphi$ was wrong in all observable time in the past, but before it has been time interval of length $m$, when $\varphi$ was true (so to say it was a local temporal knowledge).

$$
(\mathcal{M}, a) \vdash_{V} K_{2} \varphi \Leftrightarrow(\mathcal{M}, a) \vdash_{V} \square^{k} \neg \varphi \wedge \diamond^{k}(\neg \varphi \wedge \mathbf{N}(K \varphi)) .
$$

Here $K_{2}$ says that $\varphi$ was wrong in subsequent $k$ 'remembered' intervals in time, but before it has been in the past a local knowledge for a time interval of length $m$. It is easy to imagine (even being based at these simple examples) the wide possibilities for the expression properties of knowledge in time perspective (which might be achieved via the assumption that time could be non-transitive).

Now we turn to our main topic_-solution of decidability and satisfiability problems for our logic $\mathcal{L} \mathcal{L}_{N T}$. It is immediately seen that the old standard techniques to solve these problems do not work because the accessibility relation is not transitive. Therefore, the standard techniques, as rarefication (as e.g. at $[20,21]$ ), filtration, or direct usage of automatons technique, would not easily work here. Besides non-transitivity does not allow to convert formulas in more convenient forms. This is a consequence of the fact that it is impossible to say by means of formulas that two given formulas are equivalent for all past/future (it is impossible to use $\square$-time is intransitive). Therefore, we will need some preliminary work to avoid nested operations.

We will use a modernization of the technique used already by us many times (e.g. in [21]): reduction of formulas to rules and then converting them into reduced forms. This approach much simplifies all proofs since it allows to consider very simple and uniform formulas with no nested temporal operations.

Recall that a (sequential) inference rule is an expression

$$
\mathbf{r}:=\frac{\varphi_{1}\left(x_{1}, \ldots, x_{n}\right), \ldots, \varphi_{l}\left(x_{1}, \ldots, x_{n}\right)}{\psi\left(x_{1}, \ldots, x_{n}\right)},
$$

where $\varphi_{1}\left(x_{1}, \ldots, x_{n}\right), \ldots, \varphi_{l}\left(x_{1}, \ldots, x_{n}\right)$ and $\psi\left(x_{1}, \ldots, x_{n}\right)$ are formulas constructed out of letters (variables) $x_{1}, \ldots, x_{n}$. Meaning of $\mathbf{r}$ is: $\psi\left(x_{1}, \ldots, x_{n}\right)$ (which is called conclusion) follows (logically follows) from assumptions $\varphi_{1}\left(x_{1}, \ldots, x_{n}\right), \ldots, \varphi_{l}\left(x_{1}, \ldots, x_{n}\right)$. 


\section{Non-transitive LTL and logical knowledge operations}

\section{DEFINITION 5}

A rule $\mathbf{r}$ is said to be valid in a model $\mathcal{M}$ if and only if the following holds:

$$
\left[\forall a\left((\mathcal{M}, a) \vdash_{V} \bigwedge_{1 \leq i \leq l} \varphi_{i}\right)\right] \Rightarrow\left[\forall a\left((\mathcal{M}, a) \vdash_{V} \psi\right)\right]
$$

Otherwise we say $\mathbf{r}$ is refuted in $\mathcal{M}$, or refuted in $\mathcal{M}$ by $V$, and write $\mathcal{M} \nVdash_{V} \mathbf{r}$. A rule $\mathbf{r}$ is valid in a frame $\mathcal{F}$ (notation $\mathcal{F} \Vdash \mathbf{r}$ ) if it is valid in any model based at $\mathcal{F}$.

For any formula $\varphi$, we can transform $\varphi$ into the rule $x \rightarrow x / \varphi$ and employ a technique of reduced normal forms for inference rules as follows. We start from self-evident

LEMMA 6

For any formula $\varphi, \varphi$ is a theorem of $\mathcal{L} \mathcal{T} \mathcal{L}_{N T}$ (i.e. $\left.\varphi \in \mathcal{L} \mathcal{T} \mathcal{L}_{N T}\right)$ iff the rule $(x \rightarrow x / \varphi)$ is valid in any frame $\mathcal{F}$.

DEFINITION 7

A rule $\mathbf{r}$ is said to be in reduced normal form if $\mathbf{r}=\varepsilon / x_{1}$ where

$$
\varepsilon:=\bigvee_{1 \leq j \leq l}\left[\bigwedge_{1 \leq i \leq n} x_{i}^{t(j, i, 0)} \wedge \bigwedge_{1 \leq i \leq n}\left(\mathbf{N} x_{i}\right)^{t(j, i, 1)} \wedge \bigwedge_{1 \leq i, k \leq n, i \neq k}\left(x_{i} \mathbf{U} x_{k}\right)^{t(j, i, k, 1)}\right],
$$

$t(j, i, z), t(j, i, k, z) \in\{0,1\}$ and, for any formula $\alpha$ above, $\alpha^{0}:=\alpha, \alpha^{1}:=\neg \alpha$.

DEFINITION 8

Given a rule $\mathbf{r}_{\mathbf{n f}}$ in reduced normal form, $\mathbf{r}_{\mathbf{n f}}$ is said to be a normal reduced form for a rule $\mathbf{r}$ iff, for any frame $\mathcal{F}$ for $\mathcal{L} \mathcal{T} \mathcal{L}_{N T}$,

$$
\mathcal{F} \Vdash \mathbf{r} \Leftrightarrow \mathcal{F} \Vdash \mathbf{r}_{\mathbf{n f}} .
$$

THEOREM 9

There exists an algorithm running in (single) exponential time, which, for any given rule $\mathbf{r}$, constructs its normal reduced form $\mathbf{r}_{\mathbf{n f}}$.

Proof of the similar statements for various logics been suggested by us quite a while ago (e.g. it is a literal repetition of the proof for the logic $\mathcal{L} \mathcal{T} \mathcal{L}$ itself (for instance, cf. Lemma 5 in [3]).

Summarizing, a formula $\varphi$ is a theorem of $\mathcal{L} \mathcal{T} \mathcal{L}_{N T}$ (i.e. $\varphi \in \mathcal{L} \mathcal{T} \mathcal{L}_{N T}$ ) iff the rule $r:=p \rightarrow p / \varphi$ is valid at all frames (cf. Lemma 6) iff its reduced form $\mathbf{r}_{\mathbf{n f}}$ is valid at all frames (cf. Theorem 9). So, later we may work only with rules in reduced form.

\section{Main part, deciding algorithms}

Here we will need a simple modification of models for $\mathcal{L} \mathcal{T} \mathcal{L}_{N T}$ introduced earlier. As earlier let $\mathcal{F}:=\left\langle N, \leq\right.$, Next, $\left.\left(\bigcup_{i \in N}\left\{R_{i}\right\}\right)\right\rangle$, where the set of all natural numbers $N$ is the union of the intervals $\left[i, m_{i}\right]$, where any $i$ is a member of a fixed for this frame set $X$ of natural numbers, $m_{i}$ strongly bigger than $i$ and belongs to $X$. Besides, any two different $\left(i, m_{i}\right)$ have empty intersection. For any $i$ from $X$ and $j$ from $\left[i, m_{i}\right], R_{j}$ is the standard linear order on $\left[j, m_{i}\right]$. We fix notation $t(i)=m_{i}$ for any $i$ from $X$.

For any number $k$, and any $i$ from $X$ in the sequel, $t^{k}(i)$ means the result of application of function $t k$-times to the number $i$. If $a$ Next $b$ we will also write $\operatorname{Next}(a)=b$, that often will be convenient for 
our writings . For any natural number $r$, consider the following frame $\mathcal{F}(N(r))$ based at the initial interval of the frame $\mathcal{F}$ :

$$
\mathcal{F}(N(r)):=\left\langle N(r), \leq, \operatorname{Next},\left(\bigcup_{i \in N}\left\{R_{i}\right\}\right)\right\rangle,
$$

where $r>g \geq t^{2}(0)$, the base set $N(r)$ of this frame is

$$
N(r):=[0, t(0)] \cup\left[t(0), t^{2}(0)\right] \cup \cdots \cup\left[t^{g}(0), t^{g+1}(0)\right] \cup, \ldots, \cup\left[t^{r}(0), t^{r+1}(0)\right],
$$

where the relations $R_{i}$ and Next act on this frame exactly as at $\mathcal{F}$ but (i) $\operatorname{Next}\left(t^{r+1}(0)\right):=t^{g}(0)$ and (ii) Next acts on $\left[t^{r}(0), t^{r+1}(0)\right]$ as if the next interval for $\left[t^{r}(0), t^{r+1}(0)\right]$ would be $\left[t^{g}(0), t^{g+1}(0)\right]$. The valuation $V$ on such finite frame might be defined as before, and we may extend it to formulas with $\mathbf{U}$ and $\mathbf{N}$ similar as before.

LEMMA 10

For any given rule $\mathbf{r}_{\mathbf{n f}}$ in reduced normal form, if $\mathbf{r}_{\mathbf{n f}}$ is refuted in a frame $\mathcal{F}$ then $\mathbf{r}_{\mathbf{n f}}$ can be refuted in some finite model $\mathcal{F}(N(r)$ ) (where $r \in N$ ) by a valuation $V$ where the size of the frame $\mathcal{F}(N(r)$ ) is effectively computable from the size of the rule of $\mathbf{r}_{\mathbf{n f}}$ (is at most $\left[(n * l) * l^{(n * l)} *(n * l) !\right]+l^{(n * l)}$, where $l$ is the number of disjuncts in $\mathbf{r}_{\mathbf{n f}}$ and $n$ is the number of its letters).

PROOF. Let $\mathbf{r}_{\mathbf{n f}}=\varepsilon / x_{1}$ where $\varepsilon=\bigvee_{1 \leq j \leq l} \theta_{j}$,

$$
\begin{gathered}
\theta_{j}=\left[\bigwedge_{1 \leq i \leq n} x_{i}^{t(j, i, 0)} \wedge \bigwedge_{1 \leq i \leq n}\left(\mathbf{N} x_{i}\right)^{t(j, i, 1)} \wedge \bigwedge_{1 \leq i, k \leq n, i \neq k}\left(x_{i} \mathbf{U} x_{k}\right)^{t(j, i, k, 1)}\right], \\
\mathcal{F}:=\left\langle N, \leq, \operatorname{Next},\left(\bigcup_{i \in N}\left\{R_{i}\right\}\right)\right\rangle,
\end{gathered}
$$

is a frame as above and our rule is refuted in $\mathcal{F}$ by a valuation $V$.

Our first step is to show that we may take as $\mathcal{F}$ some frame where all $\left(m_{i}-i\right)$ for all $i$ from $X$ are effectively bounded. Take the original model based on $\mathcal{F}$ and refuting $\mathbf{r}_{\mathbf{n f}}$ by $V$. For any model refuting $\mathbf{r}_{\mathbf{n f}}$ by a valuation $V$ and any $i \in N$ we denote by $\theta(i)$ the unique disjunct $\theta_{j}$ from $\mathbf{r}_{\mathbf{n f}}$, which is valid at $i$ w.r.t. $V$. We will use the following notation:

$$
\begin{gathered}
\operatorname{Node}(\mathcal{F}):=\left\{k \mid k \in X, N=\bigcup_{k \in N_{1}}\left[k, m_{k}\right],\right. \\
\left.\forall k, k_{1} \in X_{1}\left[\left(k \neq k_{1}\right) \Rightarrow\left(k, m_{k}\right) \cap\left(k_{1}, m_{k_{1}}\right)=\emptyset\right]\right\} .
\end{gathered}
$$

For any $k \in X, t(k):=m_{k}, t^{r}(k)$ is $r$-times application of $t$ to $k$.

LEMMA 11

There exists a frame $\mathcal{F}_{1}$ refuting $\mathbf{r}_{\mathbf{n f}}$ by a valuation $V$, where $\forall i \in \operatorname{Node}\left(\mathcal{F}_{1}\right), m_{i}-i \leq(n * l)$, where $n$ is the number of letters in $\mathbf{r}_{\mathbf{n f}}$.

Proof. Take the original model based on $\mathcal{F}$ and refuting $\mathbf{r}_{\mathbf{n f}}$ by $V$. Consider $[0, t(0)]$. Let $a=\operatorname{Next}(0)$, take the greatest $g(a)$ in $[0, t(0)]$ (if it exists) where $\theta(a)=\theta(g(a)$ ). Delete all numbers from $[a, g(a))$ assuming $g(a)$ to be next to 0 . Let the valuation $V$ on the remaining worlds of the frame to be the same as it has been earlier. Then, the suggested modification will not effect the truth values of sub-formulas of $\mathbf{r}_{\mathbf{n f}}$ w.r.t. $V$. Hence, the resulting model based at obtained by us frame will also refute $\mathbf{r}_{\mathbf{n f}}$. 


\section{Non-transitive LTL and logical knowledge operations}

Next, take the interval $[g(a) t(0)]$ and repeat the same operations provided that the $g(g(a))$ will be inside of the interval $[0, t(0)]$. At most $l$ steps we stop, and we will obtain the frame where the first resulting interval $[0, t(0)]$ has at most $l$ elements which keep old truth values w.r.t. $V$ of all sub-formulas from the rule $\mathbf{r}_{\mathbf{n f}}$.

Consider now a frame $\mathcal{F}$ refuting $\mathbf{r}_{\mathbf{n f}}$ by $V$, were $\left[t^{k}(0), t^{k+1}(0)\right]$ is the first (smallest by $\leq$ ) interval which does not satisfy conditions of this lemma. Then we apply to $\left[t^{k}(0), t^{k+1}(0)\right]$ exactly the same transformation which we did above for $[0, t(0)]$ and immediately, as earlier, see that:

$$
t^{k}(0) \Vdash_{V} x_{k_{1}} \mathbf{U} x_{k_{2}} \text { and } t^{k}(0) \nVdash_{V} x_{k_{2}}
$$

we take first $b\left(x_{k_{1}} \mathbf{U} x_{k_{2}}\right) \in\left[t^{k}(0), t^{k+1}(0)\right]$, were $b\left(x_{k_{1}} \mathbf{U} x_{k_{2}}\right) \Vdash_{V} x_{k_{2}}$. The set $R L Z$ (realizers) of all such $b\left(x_{k_{1}} \mathbf{U} x_{k_{2}}\right)$ has at most $n$ numbers where $n$ is the set of letters from $\mathbf{r}_{\mathbf{n f}}$.

The following step of our transformation of the model based on $N$ is as follows: we, moving up, repeat to any interval $[b, c]$, where $b, c \in R L Z$ and $c$ is next to $b$ inside $R L Z$, the same operations which we applied to the interval [0,t(0)] above (keeping the accessibility relations $R_{i}$ in remaining model the same as they been earlier). The interval $\left[t^{k}(0), t^{k+1}(0)\right]$ will then have at most $n * l$ numbers. The valuation for letters of $\mathbf{r}_{\mathbf{n f}}$ in modified frame remains to be the same as earlier.

LEMMA 12

This transformation will not change the truth value of any sub-formula of $\mathbf{r}_{\mathbf{n}}$ at any world of new frame.

Using Lemma 12 and standard math induction we conclude that Lemma 11 holds.

Now, having a model $M_{1}$ at a frame of kind $\mathcal{F}_{1}$ as in Lemma 11 , refuting the rule $\mathbf{r}_{\mathbf{n f}}$, we do the following. Moving from $t(0)$ upward, starting from second interval $[t(0), t(t(0))]$, we must see the following. In some two different, not intersecting (lowermost possible) intervals $\left[t^{s}(0), t^{s+1}(0)\right]$, the strings $\left[\theta\left(t^{s}(0)\right), \ldots, \theta\left(t^{s+1}(0)\right)\right]$ coincide. Let

$$
\left[t^{s}(0), t^{s+1}(0)\right] \text { and }\left[t^{s+r}(0), t^{s+r+1}(0)\right]
$$

be these lowermost possible intervals $(s+r \geq s+1)$.

Then we delete all part $\left[t^{s+r}(0), \infty\right)$ from $\mathcal{F}_{1}$ and appoint $\left[t^{s}(0), t^{s+1}(0)\right]$ to be next interval to $\left[t^{s+r-1}(0), t^{s+r}(0)\right]$. As well we preserve the valuation $V$ to be on remaining worlds to be the same as earlier. Denote the obtained frame by $\mathcal{F}_{2}$ and the model by $\mathcal{M}_{2}$, respectively.

LEMMA 13

The truth values of all sub-formulas from $\mathbf{r}_{\mathbf{n f}}$ w.r.t. $V$ in $\mathcal{M}_{2}$ at all worlds remain the same as they have been at the original $\mathcal{F}_{1}$ w.r.t. $V$.

PROOF. It is immediate, a routine verification, because we did not change the intervals of transitivity.

Application of this lemma completes the proof for Lemma 10.

LEMMA 14

If a rule $\mathbf{r}_{\mathbf{n f}}$ in reduced normal form is refuted in a model described in Lemma 10 then $\mathbf{r}_{\mathbf{n f}}$ is not valid in $\mathcal{L} \mathcal{T} \mathcal{L}_{N T}$, i.e. there is a standard frame $\mathcal{F}$ refuting $\mathbf{r}_{\mathbf{n f}}$.

PROOF. This is a simple modification of the standard unravelling technique (it is sufficient to roll the cyclic part of the frame towards future; well, we have non-transitive accessibility relations, but nonetheless all works). 
Just in case, we give below some sufficient details. Let us have a frame of kind from Lemma 10 refuting $\mathbf{r}_{\mathbf{n f}}$ by a valuation $V$, where $\mathcal{F}(N(r)):=\left\langle N(r), \leq\right.$, Next, $\left.\left(\bigcup_{i \in N}\left\{R_{i}\right\}\right)\right\rangle$, where $r>g \geq t^{2}(0)$; $N(r):=[0, t(0)] \cup\left[t(0), t^{2}(0)\right] \cup \cdots \cup\left[t^{g}(0), t^{g+1}(0)\right] \cup, \ldots, \cup\left[t^{r}(0), t^{r+1}(0)\right]$. Recall, the relations $R_{i}$ and Next act standard way, but (i) $N \operatorname{ext}\left(t^{r+1}(0)\right):=t^{g}(0)$ and (ii) Next acts on $\left[t^{r}(0), t^{r+1}(0)\right]$ as if the next interval for $\left[t^{r}(0), t^{r+1}(0)\right]$ would be $\left[t^{g}(0), t^{g+1}(0)\right]$. Now, it is sufficient to stretch $\mathcal{F}(N(r))$ in future.

Consider the frame $\mathcal{F}(N(r, \infty)):=\left\langle W, \leq\right.$, Next, $\left.\left(\bigcup_{i \in N}\left\{R_{i}\right\}\right)\right\rangle$, where $W$ consists of the part $N(r):=[0, t(0)] \cup\left[t(0), t^{2}(0)\right] \cup \cdots \cup\left[t^{g}(0), t^{g+1}(0)\right] \cup, \ldots, \cup\left[t^{r}(0), t^{r+1}(0)\right]$ and the infinite ascending sequence of intervals

$$
\left[\left[t^{g}(0), t^{g+1}(0)\right] \cup, \ldots, \cup\left[t^{r}(0), t^{r+1}(0)\right]\right],
$$

adjoined to the standard interval $\left[0, t^{r+1}(0)\right]$. Here we do not transfer the relation Next from $\mathcal{F}(N(r))$, but assume it to act the standard way (which nonetheless acts in accordance with how it has been at $\mathcal{F}(N(r)))$. Similarly, we adjust relations Next, assuming them to act on $\left[t^{r}(0), t^{r+1}(0)\right]$, actually as in $\mathcal{F}(N(r))$, but now only towards future intervals. The valuation $V$ is transferred to $\mathcal{F}(N(r, \infty))$ and to the its extended part, being the same at copies as it has been on the originals. It is trivial routine computation to verify that all worlds and their copies will have the same truth values for sub-formulas of $\mathbf{r}_{\mathbf{n f}}$ in $\mathcal{F}(N(r, \infty))$ as they been in $\mathcal{F}(N(r))$. Lemma is proved.

Using Lemmas 10, 14, 6 and Theorem 9 we immediately derive:

\section{THEOREM 15}

Logic $\mathcal{L T}_{N T}$ is decidable; the satisfiability problem for $\mathcal{L} \mathcal{T} \mathcal{L}_{N T}$ is decidable: for any formula we can compute if it is satisfiable and if yes to compute a valuation satisfying this formula in a finite model of kind $\mathcal{F}(N(r))$.

\section{Logics with uniform bound for intransitivity, comparison}

In this short section, we would like to consider some variation of $\mathcal{L} \mathcal{T} \mathcal{L}_{N T}$-its extension, the logic generated by models with uniformly bounded measure of non-transitivity.

\section{DEFINITION 16}

A non-transitive possible-worlds linear frame $\mathcal{F}$ with uniform non-transitivity $m$ is a particular case of frames for $\mathcal{L} \mathcal{T} \mathcal{L}_{N T}$ :

$$
\mathcal{F}:=\left\langle N, \leq, \operatorname{Next},\left(\bigcup_{i \in N}\left\{R_{i}\right\}\right)\right\rangle,
$$

it is defined defined as earlier in Section4, but when $m_{i}-i \leq m$, and $m$ is a fixed for all $\mathcal{F}$ natural number (measure of intransitivity).

For any set of letters $P$ we may define an arbitrary valuation $V$ on $\mathcal{F}$ and to extend $V$ to all formulas built up from $P$ the same way as we did for $\mathcal{L} \mathcal{T} \mathcal{L}_{N T}$.

\section{DEFINITION 17}

The logic $\mathcal{L} \mathcal{L}_{N T}(m)$ is the set of all formulas which are valid at any model $\mathcal{M}$ with the measure of intransitivity $m$.

It seems that to consider and to discuss such logic is reasonable, since we may put limitations on the size of time intervals that agents (experts) may introspect in future (or to remember in past). First, immediate, easy observation about $\mathcal{L} \mathcal{T} \mathcal{L}_{N T}(m)$ is as follows: 


\section{Non-transitive LTL and logical knowledge operations}

PROPOSITION 18

Logic $\mathcal{L T}_{\mathcal{L}_{N T}}(m)$ is decidable.

Proof is trivial since for verification if a formula of temporal degree $k$ is a theorem of $\mathcal{L} \mathcal{T} \mathcal{L}_{N T}(m)$ we will need to check it on only initial part of the frames consisting only $k+1$ subsequent intervals of length at most $m$ each.

PROPOSITION 19

$\mathcal{L} \mathcal{T} \mathcal{L}_{N T} \subset \mathcal{L} \mathcal{T} \mathcal{L}_{N T}(m)$ and $\mathcal{L} \mathcal{T} \mathcal{L}_{N T}(m+1) \subset \mathcal{L} \mathcal{T} \mathcal{L}_{N T}(m)$.

Proof is trivial, it immediately follows from definitions and the observations that

$$
\begin{gathered}
\left(\bigwedge_{i \leq m} \mathbf{N}^{i} p \rightarrow \square p\right) \in \mathcal{L} \mathcal{T} \mathcal{L}_{N T}(m), \text { but } \\
\left(\bigwedge_{i \leq m} \mathbf{N}^{i} p \rightarrow \square p\right) \notin \mathcal{L} \mathcal{T} \mathcal{L}_{N T}(m+1), \text { and }\left(\bigwedge_{i \leq m} \mathbf{N}^{i} p \rightarrow \square p\right) \notin \mathcal{L} \mathcal{T} \mathcal{L}_{N T} .
\end{gathered}
$$

PROPOSITION 20

$\mathcal{L} \mathcal{T} \mathcal{L}_{N T}=\bigcap_{m \in N} \mathcal{L} \mathcal{T} \mathcal{L}_{N T}(m)$.

ProOF. This is again simple, if a formula $\varphi$ with temporal degree $k$ does not belong to $\mathcal{L} \mathcal{T} \mathcal{L}_{N T}$ than $\varphi$ is rejected in a frame in its initial part consisting of $k+1$ subsequent intervals. Then it is sufficient to take the modification of this frame consisting of this initial part and subsequent duplications of its final interval in future with arbitrary valuation of the letters of $\varphi$ at the duplications (and with arbitrary accessibility relations $R_{i}$ at the duplication part). This model again will disprove $\varphi$ and transitivity bound of its intervals of transitivity will be $m$ which is the maximal measure of the transitivity in the original $k+1$ intervals.

Summarizing our observations, decidability for $\mathcal{L} \mathcal{T} \mathcal{L}_{N T}(m)$ is trivial which is not the case for $\mathcal{L} \mathcal{T} \mathcal{L}_{N T}$ itself. Though if we would have a computable axiomatization for $\mathcal{L} \mathcal{T} \mathcal{L}_{N T}$ it would give (by Proposition 20) a worst scenario expectation deciding algorithm for $\mathcal{L} \mathcal{T} \mathcal{L}_{N T}$. But we found in this our article an effective one-with the computable upper bound for rejecting frames.

Proposition 21

$\mathcal{L} \mathcal{T} \mathcal{L} \nsubseteq \mathcal{L} \mathcal{T} \mathcal{L}_{N T}$ and $\mathcal{L} \mathcal{T} \mathcal{L} \nsubseteq \mathcal{L} \mathcal{T} \mathcal{L}_{N T}(m)$ for all $m$.

Proof is evident since $\square p \rightarrow \square \square P \in \mathcal{L} \mathcal{T} \mathcal{L}$.

PROPOSITION 22

$\mathcal{L} \mathcal{T} \mathcal{L}_{N T}(m) \nsubseteq \mathcal{L} \mathcal{T} \mathcal{L}$ for all $m$

Proof is evident since

$$
\left(\bigwedge_{i \leq m} \mathbf{N}^{i} p \rightarrow \square p\right) \in \mathcal{L} \mathcal{T} \mathcal{L}_{N T}(m) .
$$

\section{Open problems}

There are many remaining open interesting problems in suggested framework. E.g. the problem described at the final part of Section 6 (approach (v)) for multi-agent case. The problems of axiomatization for $\mathcal{L} \mathcal{T} \mathcal{L}_{N T}$ and for $\mathcal{L} \mathcal{T} \mathcal{L}_{N T}(m)$ are open. It looks reasonable to extend our approach to linear logics with linear non-transitive but continuous time. The problems of recognizing admissible 
rules for $\mathcal{L} \mathcal{T} \mathcal{L}_{N T}$ and for $\mathcal{L} \mathcal{T} \mathcal{L}_{N T}(m)$ are open yet. The multi-agent approach in the framework suggested in this article, but for the case when any $n \in N$ would be represented by a cluster (circle) with $n$ agents knowledge relations $K_{i}$, is also not investigated.

\section{References}

[1] S. Artemov. Justified common knowledge. Theoretical Computer Science, 357, 4-22, 2006.

[2] S. Artemov and E. Nogina. Introducing justification into epistemic logic. Journal of Logic and Computation, 15, 1059-1073, 2005.

[3] S. Babenyshev and V. Rybakov. Linear temporal logic LTL: basis for admissible rules. Journal of Logic and Computation, 21, 157-177, 2011.

[4] F. Belardinelli and A. Lomuscio. Interactions between knowledge and time in a first-order logic for multi-agent systems: completeness results. Journal of Artificial Intelligence Research, 45, $1-45,2012$.

[5] P. Balbiani and D. Vakarelov. A modal logic for indiscernibility and complementarity in information systems. Fundamenta Informaticae 50, 243-263, 2002.

[6] R. Fagin, J. Halpern, Y. Moses and M. Vardi. Reasoning About Knowledge, The MIT Press, 1995.

[7] H. Friedman. One hundred and two problems in mathematical logic. Journal of Symbolic Logic, 40, 113-130, 1975.

[8] D. M. Gabbay, I. M. Hodkinson and M. A. Reynolds. Temporal Logic: Mathematical Foundations and Computational Aspects, V. 1. Clarendon Press, 1994.

[9] D. M. Gabbay and I. M. Hodkinson. An axiomatization of the temporal logic with until and since over the real numbers. Journal of Logic and Computation, 1, 229-260, 1990.

[10] D. Gabbay and I. Hodkinson. Temporal logic in context of databases. In J. Copeland, editor, Logic and Reality, Essays on the legacy of Arthur Prior, Oxford University Press, 1995.

[11] J. Halpern, D. Samet and E. Segev. Defining knowledge in terms of belief. The modal logic perspective. The Review of Symbolic Logic, 2, 469-487, 2009.

[12] J. Hintikka. Knowledge and Belief: An Introduction to the Logic of the Two Notions. Cornell University Press, 1962.

[13] A. Lomuscio and J. Michaliszyn. An epistemic Halpern-Shoham logic. In Proceedings of the 23rd International Joint Conference on Artificial Intelligence (IJCAI13), pp. 1010-1016. AAAI Press, 2013,

[14] Z. Manna and A. Pnueli. The Temporal Logic of Reactive and Concurrent Systems: Specification. Springer, 1992.

[15] Z. Manna and A. Pnueli. Temporal Verification of Reactive Systems: Safety. Springer, 1995.

[16] D. McLean and V. Rybakov. Multi-agent temporary logic $T S 4_{K_{n}}^{U}$ based at non-linear time and imitating uncertainty via agents' interaction. In Artificial Intelligence and Soft Computing, Conference Proceedings, pp. 375-384. Springer, 2013.

[17] V. V. Rybakov. Refined common knowledge logics or logics of common information. Archive for mathematical Logic, 42, 179-200, 2003.

[18] V. V. Rybakov. Logical consecutions in discrete linear temporal logic. Journal of Symbolic Logic, 70, 1137-1149, 2005.

[19] V. V. Rybakov. Linear temporal logic with until and next, logical consecutions. Annals of Pure and Applied Logic, 155, 32-45, 2008.

[20] V. Rybakov. Logic of knowledge and discovery via interacting agents—Decision algorithm for true and satisfiable statements. Information Sciences, 179, 1608-1614, 2009. 
14 Non-transitive LTL and logical knowledge operations

[21] V. Rybakov. Linear temporal logic $L T L_{K_{n}}$ extended by multi-agent logic $K_{n}$ with interacting agents. Journal of logic and Computation, 19, 989-1017, 2009.

[22] V. Rybakov and S. Babenyshev. Multi-agent logic with distances based on linear temporal frames. In Artificial Intelligence and Soft Computing, Conference Proceedings, pp. 337-344. Springer, 2010.

[23] V. V. Rybakov. Chance discovery and unification in linear modal logic. In Knowledge-Based and Intelligent Information and Engineering Systems (KES 2011), LNCS, 6882, 478-485, 2011.

[24] V. V. Rybakov. Logical analysis for chance discovery in multi-agents environment. In Knowledge-Based and Intelligent Information and Engineering Systems (KES 2012), Conference Proceedings, pp. 1593-1601. Springer, 2012.

[25] V. V. Vladimir. Rybakov: A note on parametrized knowledge operations in temporal logic. CoRR abs/1405.0559 (2014).

[26] V. V. Vladimir. Rybakov: Linear non-transitive temporal logic, knowledge operations, algorithms for admissibility. CoRR abs/1406.2783 (2014).

[27] M. Vardi. An automata-theoretic approach to linear temporal logic. In Y. Banff, editor Higher Order Workshop, pp. 238-266, 1995. Available at http://citeseer.ist.psu.edu/ vardi96automatatheoretic.html.

[28] M. Y. Vardi. Reasoning about the past with two-way automata. In K. G. Larsen, S. Skyum and G. Winskel, editors. ICALP, LNCS, 1443, 628-641. Springer, 1998.

[29] D. Vakarelov. A modal characterization of indiscernibility and similarity relations in Pawlak's information systems. RSFDGrC, 1, 12-22, 2005.

[30] M. Wooldridge and A. Lomuscio. Multi-agent VSK logic. In Proceedings of the Seventh European Workshop on Logics in Artificial Intelligence (JELIAI-2000). Springer, 2000.

[31] M. Wooldridge. An automata-theoretic approach to multiagent planning. In Proceedings of the First European Workshop on Multiagent Systems (EUMAS 2003), Oxford University, 2003.

[32] M. Wooldridge, M.-P. Huget, M. Fisher and S. Parsons. Model checking multi-agent systems: The MABLE language and its applications. International Journal on Artificial Intelligence Tools, 15, 195-225, 2006.

Received 30 March 2015 